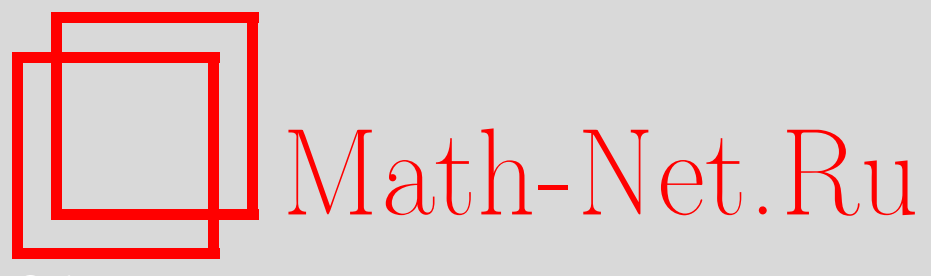

А. А. Панчишкин, Две гипотезы о модулярном подъеме семейств зигелевых модулярных форм, Матем. заметки, 2010, том 88, выпуск 4, 565-574

DOI: https://doi.org/10.4213/mzm8853

Использование Общероссийского математического портала Math-Net.Ru подразумевает, что вы прочитали и согласны с пользовательским соглашением http://www . mathnet.ru/rus/agreement

Параметры загрузки:

IP : 54.210 .77 .194

26 апреля 2023 г., 13:58:43

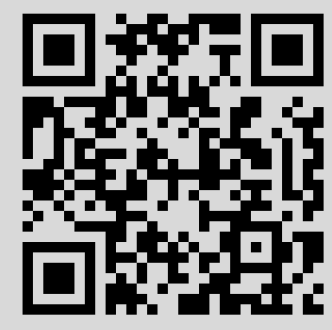


Том 88 выпуск 4 октябрь 2010

УДК 511.333

\section{Две гипотезы о модулярном подъеме семейств зигелевых модулярных форм}

\section{А. А. Панчишкин}

Для простого числа $p$ и положительного целого числа $n$ изучаются методы построения $p$-адических семейств зигелевых модулярных форм рода $n$ посредством использования некоторых отображений подъема. Дается описание $L$-функций, связанных с зигелевыми модулярными формами, а также их аналитических свойств. Формулируются две гипотезы о модулярном подъеме семейств зигелевых модулярных форм и о существовании отображений подъема из $\mathrm{GSp}_{r} \times \mathrm{GSp}_{2 m}$ в $\mathrm{GSp}_{r+2 m}$ для некоторых положительных чисел $r$ и $m$.

Библиография: 20 названий.

Памяти Анатолия Алексеевича Карачубы

\section{1. Введение}

В данной работе рассматриваются дзета-функции зигелевых модулярных форм и их $p$-адические свойства, развивающие эту тему в новом контексте $p$-адических семейств.

Рассмотрим зигелеву модулярную группу $\Gamma_{n}=\operatorname{Sp}_{n}(\mathbb{Z}) \subset \mathrm{SL}_{2 n}(\mathbb{Z})$ рода $n$, и пусть $p$ - фиксированное простое число.

Здесь для любого коммутативного кольца $R$ используется обозначение

$$
\operatorname{Sp}_{n}(R)=\left\{M=\left(\begin{array}{cc}
A & B \\
C & D
\end{array}\right) \in \mathrm{SL}_{2 n}(R) \mid{ }^{t} M J_{n} M=J_{n}\right\}, \quad J_{n}=\left(\begin{array}{cc}
0_{n} & -I_{n} \\
0_{n} & J_{n}
\end{array}\right),
$$

для симплектической группы рода $n$, которая является алгебраической подгруппой в группе

$$
\operatorname{GSp}_{n}(R)=\left\{M=\left(\begin{array}{cc}
A & B \\
C & D
\end{array}\right) \in \mathrm{GL}_{2 n} \mid{ }^{t} M J_{n} M=\mu J_{n}, \mu \in R^{*}\right\}
$$

симплектических подобий.

Работа выполнена при поддержке Института Фурье (Гренобль, Франция), Университета Пурдью (Лафайетт, США), и Лаборатории Понселе (Москва) во время визитов автора в Москву в феврале-июле 2009 г., в Кингс Колледж (Лондон) в июне 2009 г., в Университет Пурдью (Лафайетт, США) в июле 2009 г.

(C) А.А. ПАнчишкин, 2010 
Пусть также символ $\mathscr{M}_{k}\left(\Gamma_{n}\right)$ обозначает векторное пространство голоморфных зигелевых модулярных форм относительно группы $\Gamma_{n}$, и $\mathscr{S}_{k}\left(\Gamma_{n}\right)$ обозначает векторное подпространство голоморфных зигелевых параболических форм.

В данной работе обсуждаются следующие темы:

1) семейства зигелевых модулярных форм и их $L$-функций;

2) аналитические свойства $L$-функций относительно группы $\mathrm{Sp}_{n}$;

3) построение $p$-адических семейств зигелевых модуляных форм, параметризованных весом $k$ с использованием конструкций Икеды-Мияваки;

4) два вида модулярного подъема из $\mathrm{GSp}_{r} \times \mathrm{GSp}_{2 m}$ в $\mathrm{GSp}_{r+2 m}($ рода $n=r+2 m)$.

Знаменитые примеры $p$-адических семейств эллиптических модулярных форм (для рода $n=1)$. Рассматриваются семейства

$$
\begin{aligned}
G_{k} & =\frac{\zeta(1-k)}{2}+\sum_{n \geqslant 1} \sigma_{k-1}(n) q^{n} & & \text { (ряды Эйзенштейна веса } k \geqslant 4) \\
\varphi_{k} & =\sum_{\mathfrak{a} \subset \mathscr{O}_{K}} \lambda^{k-1}(\mathfrak{a}) q^{N \mathfrak{a}} & & \text { (параболические формы СМ-типа веса } k \geqslant 2) .
\end{aligned}
$$

Семейства $\varphi_{k}$ были изучены Маниным и Вишиком [1], а также Катцом [2]. Семейства Эйзенштейна (1.1) были применены Серром [3] с целью модулярного объяснения сравнений Куммера для чисел Бернулли

$$
\zeta(1-k)=-\frac{B_{k}}{k}
$$

для нового построения $p$-адической дзета-функции Куботы-Леопольдта.

Семейства (1.2) СМ-типа отвечают степеням грессен-характера $\lambda$ для мнимого квадратичного поля $K$ (соответственно грессен-характера типа $A_{0}$ группы классов иделей $\mathbb{A}_{K^{*}} / K^{*}$ (в смысле Вейля [4])) для поля $K$, причем $L(E, s)=L(\lambda, s)$ для некоторой эллиптической кривой $E$ плексным умножением на элементы поля $K$ (см. Дойринг [5]), причем функции $\varphi_{k}$ выражались через некоторые тэта-ряды со сферическими многочленами, что доказывает модулярность эллиптической кривой $E$ с комплексным умножением, причем

$$
L(E, s)=L(\lambda, s)=L\left(\varphi_{2}, s\right) .
$$

Семейства $\varphi_{k}$ СМ-типа (1.2) доставляют также пример обыкновенных семейств в смысле теории Хиды (см. [6]) в случае простого числа $p$, pacnaдающегося в поле $K$.

Как было доказано Маниным и Вишиком [1], функции двух переменных, заданные нормализованными специальными значениями $L^{*}\left(\varphi_{k}, s\right)$ для $L$-функций, отвечающих семейству $\varphi_{k}$,

$$
(s, k) \mapsto L^{*}\left(\varphi_{k}, s\right), \quad s=1, \ldots, k-1,
$$

допускают продолжение до $p$-адической аналитической функции двух $p$-адических переменных $(s, k)$. 
От эллиптических модулярных форм к зигелевым модулярным формам. Теперь рассмотрим зигелеву модулярную форму

$$
f=\sum_{\mathscr{T} \in B_{n}} a_{\mathscr{T}} q^{\mathscr{T}} \in \mathscr{M}_{k}\left(\Gamma_{n}\right)
$$

веса $k$ и рода $n$ на зигелевой полуплоскости

$$
\mathbb{H}_{n}=\left\{{ }^{t} z=z \in \mathrm{M}_{n}(\mathbb{C}) \mid \operatorname{Im}(z)>0\right\}
$$

где

$$
\begin{gathered}
q^{\mathscr{T}}=\exp (2 \pi \sqrt{-1} \operatorname{tr}(\mathscr{T} z)), \\
B_{n}=\left\{{ }^{t} \mathscr{T}=\mathscr{T} \in \mathrm{M}_{n}(\mathbb{Q}) \mid \mathscr{T} \geqslant 0-\text { полуцелые матрицы }\right\} .
\end{gathered}
$$

Для простоты будем рассматривать в данной работе только скалярнозначные зигелевы модулярные формы.

Предположим, что $f \in \mathscr{M}_{k}\left(\Gamma_{n}\right)$ является собственной функцией всех операторов Гекке. Тогда спинорная $L$-функция $L(f, \operatorname{Spin}, s)$ и стандартная $L$-функция $L(F, \operatorname{St}, s)$ для $f($ при $s \in \mathbb{C})$ суть произведения

$$
L(f, \text { Spin, } s)=\prod_{p} Q_{F, p}\left(p^{-s}\right)^{-1}, \quad L(f, S t, s)=\prod_{p} R_{f, p}\left(p^{-s}\right)^{-1}
$$

где многочлены $Q_{f, p}(X)$ и $R_{f, p}(X)$ являются обобщенными многочленами Гекке в $p$-степени $2^{n}$ и $2 n+1$, определение которых приведено при формулировке результатов.

Вопрос 1. Построить $p$-адические семейства

$$
k \mapsto f_{k}=\sum_{\mathscr{T} \in B_{n}} a_{\mathscr{T}}(k) q^{\mathscr{T}} \in \mathscr{M}_{k}\left(\Gamma_{n}\right)
$$

зигелевых модулярных форм $f_{k}$ веса $k$ и рода $n$, где коэффициенты Фурье $a_{\mathscr{T}}(k)$ формы $F_{k}$ даются некоторыми $p$-адическими аналитическими функциями $k \mapsto a_{\mathscr{T}}(k)$ для $((\operatorname{det} 2 \mathscr{T}), p)=1$.

Вопрос 2. Распространить конструкции $p$-адических $L$-функций на нормализованные собственные значения $L$-функций

$$
(s, k) \mapsto L^{*}\left(f_{k}, \text { Spin, } s\right), \quad(s, k) \mapsto L^{*}\left(f_{k}, \text { St }, s\right) .
$$

Отметим, что вопрос 2 о p-адической интерполяции специальных значений рассматриваемых $L$-функций тесно связан с вопросом 1 в силу общего феномена: линейная форма, задающая нормализованное специальное значение $L$-функции, может быть выражена через линейные формы, задаваемые коэффициентами Фурье. Более того, этот феномен оказывается применимым в подходящих семействах модулярных форм.

Однако, вопрос 2 более деликатен; частичные ответы на него будут развиты в последующих работах. 


\section{2. $L$-функции зигелевых модулярных форм}

Разложение Фурье зигелевой модулярной формы. Пусть

$$
f=\sum_{\mathscr{T} \in B_{n}} a(\mathscr{T}) q^{\mathscr{T}} \in \mathscr{M}_{k}\left(\Gamma_{n}\right)
$$

- зигелева модулярная форма веса $k$ и рода $n$ на зигелевой верхней полуплоскости

$$
\mathbb{H}_{n}=\left\{{ }^{t} z=z \in \mathrm{M}_{n}(\mathbb{C}) \mid \operatorname{Im}(z)>0\right\} .
$$

Напомним основные факты о разложении Фурье формы $f$, используя символ

$$
q^{\mathscr{T}}=\exp (2 \pi \sqrt{-1} \operatorname{tr}(\mathscr{T} z))=\prod_{i=1}^{n} q_{i i}^{\mathscr{T}_{i i}} \prod_{i<j} q_{i j}^{2 \mathscr{I}_{i j}} \in \mathbb{C} \llbracket q_{11}, \ldots, q_{n n} \rrbracket\left[q_{i j}, q_{i j}^{-1}\right]_{i, j=1, \ldots, m},
$$

где $q_{i j}=\exp \left(2 \pi\left(\sqrt{-1} z_{i, j}\right)\right), \mathscr{T}$ - матрица из полугруппы $B_{n}$.

Операторы Гекке и сферическое отображение. Напомним, что локальная $p$-алгебра Гекке относительно пары $\left.\left(\operatorname{Sp}_{n}(\mathbb{Z}), \operatorname{GSp}_{n}\left(\mathbb{Z}\left[p^{-1}\right]\right)\right)\right)$, обозначаемая

$$
\mathscr{L}_{n, p}=\mathbb{Q}\left[\mathbf{T}(p), \mathbf{T}_{1}\left(p^{2}\right), \ldots, \mathbf{T}_{n}\left(p^{2}\right)\right],
$$

порождена следующими $n+1$ операторами Гекке:

$$
\begin{aligned}
\mathbf{T}(p) & :=T(\underbrace{1, \ldots, 1}_{n}, \underbrace{p, \ldots, p}_{n}), \\
\mathbf{T}_{i}\left(p^{2}\right) & :=T(\underbrace{1, \ldots, 1}_{n-i}, \underbrace{p, \ldots, p}_{i}, \underbrace{p^{2}, \ldots, p^{2}}_{n-i}, \underbrace{p, \ldots, p}_{i}), \quad i=1, \ldots, n,
\end{aligned}
$$

а сферическое отображение

$$
\Omega: \mathscr{L}_{n, p}=\mathbb{Q}\left[\mathbf{T}(p), \mathbf{T}_{1}\left(p^{2}\right), \ldots, \mathbf{T}_{n}\left(p^{2}\right)\right] \rightarrow \mathbb{Q}\left[x_{0}, x_{1}, \ldots, x_{n}\right]
$$

задается некоторым гомоморфизмом колец (см. [7]).

Здесь для любого $m \in \mathbb{N} T\left(a_{1}, \ldots, a_{m}\right)$ обозначает двойной класс диагональной матрицы $\operatorname{diag}\left(a_{1}, \ldots, a_{m}\right)$ с элементами $a_{1}, \ldots, a_{m}$ на диагонали, так что мы полагаем $m=2 n$ для группы $\mathrm{GSp}_{n}$ и $m=n$ для группы $\mathrm{GL}_{n}$. Напомним, что алгебра Гекке $\mathscr{L}_{n, p}$ действует на пространствах модулярнах форм и на их подпространствах параболических форм.

Параметры Сатаке собственных функций операторов Гекке. Предположим, что $f \in \mathscr{M}_{k}\left(\Gamma_{n}\right)$ - собственная функция всех операторов Гекке $f \mapsto f \mid T$, $T \in \mathscr{L}_{n, p}$, для всех простых чисел $p$, так что $f \mid T=\lambda_{f}(T) f$. Тогда каждое число $\lambda_{f}(T) \in \mathbb{C}$ определяет гомоморфизм $\lambda_{f}: \mathscr{L}_{n, p} \rightarrow \mathbb{C}$, задаваемый набором $n+1$ комплексных чисел ( определенных с точностью до действия группы Вейля $W_{n}$, так что

$$
\lambda_{f}(T)=\Omega(T)\left(\alpha_{0}, \alpha_{1}, \ldots, \alpha_{n}\right) .
$$


В частности,

$$
\begin{aligned}
\lambda_{f}([\mathbf{p}]) & =\alpha_{0}^{2} \alpha_{1} \cdots \alpha_{n}=p^{k n-n(n+1) / 2} \\
\lambda_{f}(\mathbf{T}(p)) & =\Omega(\mathbf{T}(p))\left(\alpha_{0}, \alpha_{1}, \ldots, \alpha_{n}\right) \\
& =\alpha_{0}\left(1+\alpha_{1}\right) \cdots\left(1+\alpha_{n}\right)=\sum_{j=0}^{n} \alpha_{0} s_{j}\left(\alpha_{1}, \alpha_{2}, \ldots, \alpha_{n}\right),
\end{aligned}
$$

где

$$
[\mathbf{p}]=[\mathbf{p}]_{n}=\mathbf{T}_{n}\left(p^{2}\right)=T(\underbrace{p, \ldots, p}_{2 n})=p \mathbf{I}_{2 n} .
$$

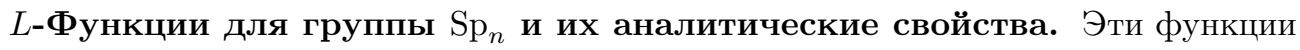
определяются как эйлеровы произведения с $p$-множителями

$$
\begin{aligned}
& Q_{f, p}(X)=\left(1-\alpha_{0} X\right) \prod_{r=1}^{n} \prod_{1 \leqslant i_{1}<\cdots<i_{r} \leqslant n}\left(1-\alpha_{0} \alpha_{i_{1}} \cdots \alpha_{i_{r}} X\right), \\
& R_{f, p}(X)=(1-X) \prod_{i=1}^{n}\left(1-\alpha_{i}^{-1} X\right)\left(1-\alpha_{i} X\right) \in \mathbb{Q}\left[\alpha_{0}^{ \pm 1}, \ldots, \alpha_{n}^{ \pm 1}\right][X] .
\end{aligned}
$$

Спинорная $L$-функция $L(f, \operatorname{Spin}, s)$ и стандартная $L$-функция $L(f, \operatorname{St}, s)$ формы $f$ (для $s \in \mathbb{C}$ с $\operatorname{Re}(s) \gg 0$ ) определены равенствами

$$
L(f, \text { Spin }, s)=\prod_{p} Q_{f, p}\left(p^{-s}\right)^{-1}, \quad L(f, \mathrm{St}, s)=\prod_{p} R_{f, p}\left(p^{-s}\right)^{-1} .
$$

Аналитические свойства спинорных $L$-функций $L(f$,Spin, $s)$ были изучены Гекке [8] $(n=1)$, Андриановым [7] $(n=2)$, а также Асгари-Шмидтом [9], где теория эйлеровых произведений Ленглендса была применена к подгруппе Леви исключительной группы типа $F_{4}$, и применена для построения мероморфного продолжения спинорной $L$-функции для рода $n=3$.

Аналитические свойства стандартных $L$-функций $L(f, \mathrm{St}, s)$ были изучены Ранкином [10] и Шимурой [11] для $n=1$.

Андрианов и Калинин [12] рассмотрели случай четного рода $n$, а Бёхерер [13] распространил эти результаты на случай произвольного рода, используя другой подход. Для $n=1$ и нормализованной параболической собственной формы

$$
f(z)=\sum_{n=0}^{\infty} a(n) e(n z) \in \mathscr{M}_{k}\left(\Gamma_{1}\right)
$$

мы имеем равенство

$$
L(f, \text { St }, s)=L_{2, f}(s+k-1)
$$

где

$$
L_{2, f}(s)=\zeta(2 s-2 k+2) \sum_{n=1}^{\infty} a\left(n^{2}\right) n^{-s}
$$

обозначает симметрический квадрат модулярной формы $f$ (см. [11]). 


\section{3. Подъемы Икеды зигелевых модулярных форм}

Напомним конструкции Икеды [14]-[16]: пусть $k$ - четное положительное число, $\varphi \in S_{2 k}\left(\Gamma_{1}\right)$ - нормализованная параболическая собственная форма веса $2 k$, $g:=F_{2 m}(\varphi) \in S_{k+m}\left(\Gamma_{2 m}\right)$ - подгем Дьюка-Имамоглу-Икеды формы $\varphi ;$ этот подъем рода $2 m$ (при предположении $k \equiv m \bmod 2, m \in \mathbb{N}$ ). Оказывается, что этот подъем допускает функториальную характеризацию посредством равенства $L$-функций (см. [14]):

$$
L(g, \mathrm{St}, s)=L\left(F_{2 m}(\varphi), \mathrm{St}, s\right)=\zeta(s) \prod_{i=1}^{2 m} L(\varphi, s+k+m-i) .
$$

p-Адические версии конструкций Икеды. Теперь рассмотрим $p$-адическое семейство

$$
k \mapsto \varphi_{2 k}=\sum_{n=1}^{\infty} a_{n}(2 k) q^{n} \in \overline{\mathbb{Q}} \llbracket q \rrbracket \subset \mathbb{C}_{p} \llbracket q \rrbracket,
$$

где коэффициенты Фурье $a_{n}(2 k)$ нормализованной параболической собственной формы $\varphi_{2 k}$, а также один из $p$-параметров Сатаке $\alpha(2 k):=\alpha_{p}(2 k)$ даются некоторыми $p$-адическими аналитическими функциями $k \mapsto a_{n}(2 k)$ для $(n, p)=1$ и $k \mapsto \alpha_{p}(2 k)$.

Тогда оказывается, что разложения Фурье модулярных форм $F=F_{2 m}\left(\varphi_{2 k}\right)$ могут быть явно вычислены и что эти зигелевы модулярные формы дают примеры p-адических семейств зигелевых модулярных форм (при определенных условиях сравнения на $k, m$ и $r)$.

Для точной формулировки результата рассмотрим $p$-адическое семейство $\left\{\varphi_{2 k}\right\}$ эллиптических модулярных форм $\left\{\varphi_{2 k}\right\}$ четного веса $2 k$ и уровня 1 . При этом не делается предположения о том, является ли семейство $\left\{\varphi_{2 k}\right\}$ обыкновенным в смысле Хиды или нет.

Теорема (Хиса-Аки Кавамура, Панчишкин). После подходящей регуляризации семейство

$$
g_{k+m}^{2 m}:=F_{2 m}\left(\varphi_{2 k}\right) \in S_{k+m}\left(\Gamma_{2 m}\right)
$$

является р-адическим семейством зигелевых модулярных форм веса $k+m$ и рода $2 m$.

Красивые численные примеры для этой теоремы были вычислены недавно Кавамурой [17] для определенных форму Рамануджана $\Delta(z)=\varphi_{12}(z)$, а также для семейств, содержащих собственные параболические формы $f_{18}$ и $f_{20}$ для простых чисел $p=11, p=19, p=23$. (Эти примеры были сообщены автору в частной беседе в июле 2008 г.)

\section{4. Голоморфные модулярные подъемы}

Две гипотезы о голоморфных модулярных подъемах из $\mathrm{GSp}_{r} \times \mathrm{GSp}_{2 m}$ в $\mathrm{GSp}_{r+2 m}$ (рода $\left.n=r+2 m\right)$. Обсудим подъемы из $\mathrm{GSp}_{r} \times \mathrm{GSp}_{2 m}$ в $\mathrm{GSp}_{r+2 m}$ рода $n=r+2 m$. 
ГипотезА I (о подъеме первого рода из $\mathrm{GSp}_{r} \times \mathrm{GSp}_{2 m}$ в $\mathrm{GSp}_{r+2 m}$ ). Пусть

$$
(f, g) \in \mathscr{M}_{k+m}\left(\Gamma_{r}\right) \times \mathscr{M}_{k+r+m}\left(\Gamma_{2 m}\right)
$$

- пара собственных параболических форм относительно алгебр Гекке $\mathscr{L}_{r}=\bigotimes_{p} \mathscr{L}_{r, p}$ u $\mathscr{L}_{2 m}=\bigotimes_{p} \mathscr{L}_{2 m, p}$. Обозначим через $\alpha_{0}, \alpha_{1}, \ldots, \alpha_{r} u \beta_{0}, \beta_{1}, \ldots, \beta_{2 m}$ p-параметръ Сатаке форм $f$ u $g$ (напомним, что эти параметры определены с точностью до действия группы Вейля).

Тогда существует собственная параболическая форма $F$ относительно алгебрь Гекке $\mathscr{L}_{r+2 m}$ рода $r+2 m$ и веса $k$ с параметрами Сатаке

$$
\begin{aligned}
\gamma_{0}=\alpha_{0} \beta_{0}, \quad \gamma_{1} & =\beta_{1}, \quad \gamma_{2}=\beta_{2}, \quad \ldots, \quad \gamma_{2 m}=\beta_{2 m}, \\
\gamma_{2 m+1} & =\alpha_{1}, \quad \ldots, \quad \gamma_{r+2 m}=\alpha_{r},
\end{aligned}
$$

для подходящего выбора р-параметров Сатаке $\alpha_{0}, \alpha_{1}, \ldots, \alpha_{r} u \beta_{0}, \beta_{1}, \ldots, \beta_{2 m}$ борм $f$ $u g$.

Свидетельство в пользу этой гипотезы происходит из конструкций Икеды-Мияваки [14]-[16]: пусть $k$ - положительное четное число, $\varphi \in S_{2 k}\left(\Gamma_{1}\right)$ - нормализованная собственная форма веса $2 k, g:=F_{2 m}(\varphi) \in S_{k+m}\left(\Gamma_{2 m}\right)$ - подъем Дьюка-Имамоглу-Икеды формы $\varphi$; напомним, что этот подъем рода $2 m$ (при предположении $k \equiv$ $m \bmod 2, m \in \mathbb{N})$.

Рассмотрим далее произвольную собственную зигелеву параболическую форму рода $r$ и веса $k+m+r$ с $m, r \geqslant 1$.

Рассмотрим далее подъем Икеды $F_{2 m+2 r}(\varphi) \in S_{k+m+r}\left(\Gamma_{2 m+2 r}\right)$ и интеграл

$$
\mathscr{F}_{\varphi, f}=\int_{\Gamma_{r} \backslash \mathbb{H}_{r}} F_{2 m+2 r}(\varphi)\left(\left(\begin{array}{ll}
z & \\
& z^{\prime}
\end{array}\right)\right) f\left(z^{\prime}\right)\left(\operatorname{det} \operatorname{Im}\left(z^{\prime}\right)\right)^{k+m+r-1} d z^{\prime} \in S_{k+m+r}\left(\Gamma_{2 m+r}\right),
$$

определяющий подбем Икедъ-Мияваки, где $z \in \mathbb{H}_{2 m+r}, z^{\prime} \in \mathbb{H}_{r}$.

При условии необращения в нуль интеграла $\mathscr{F} \varphi, f$ справедливо равенство

$$
L\left(\mathscr{F}_{\varphi, f}, \mathrm{St}, s\right)=L(f, \mathrm{St}, s) \prod_{i=1}^{2 m} L(\varphi, s+k+m+r-i) .
$$

Пусть далее $2 k$ - четное положительное число, $\varphi \in S_{2 k}\left(\Gamma_{1}\right)$ - нормализованная собственная форма веса $2 k, F_{2 m} \in S_{k+m}\left(\Gamma_{2 m}\right)$ - подъем Икеды формы $\varphi$ рода $2 m$ (предполагается, что $k \equiv m \bmod 2, m \in \mathbb{N}$ ).

Теперь пусть $f \in S_{k+m+r}\left(\Gamma_{r}\right)$ обозначает произвольную собственную форму рода $r$ и веса $k+m+r$ с $m, r \geqslant 1$.

Тогда пример выполнимости этой версии гипотезы дается следующим отображением:

$$
\begin{aligned}
& (f, g)=\left(f, F_{2 m}(\varphi)\right) \mapsto \mathscr{F}_{\varphi, f} \in S_{k+r+m}\left(\Gamma_{r+2 m}\right), \\
& (f, g)=\left(f, F_{2 m}(\varphi)\right) \in S_{k+r+m}\left(\Gamma_{r}\right) \times S_{k+m}\left(\Gamma_{2 m}\right) .
\end{aligned}
$$

Еще один пример выполнимости этой гипотезы приходит из рядов Зигеля-Эйзенштейна

$$
f=E_{k+m+r}^{r}, \quad g=E_{k+m}^{2 m}
$$


родов $r$ и $2 m$ и весов $k+m+r$ и $k+m$ соответственно: мы имеем

$$
\begin{array}{llll}
\alpha_{0}=1, & \alpha_{1}=p^{k+m+r-r}, & \ldots, & \alpha_{r}=p^{k+m+r-1}, \\
\beta_{0}=1, & \beta_{1}=p^{k+m-2 m}, & \ldots, & \beta_{2 m}=p^{k+m-1},
\end{array}
$$

откуда видно, что

$$
\begin{array}{ccc}
\gamma_{0}=1, \quad \gamma_{1}=\beta_{1}=p^{k+r+m-(r+2 m)}, & \ldots, \quad \gamma_{2 m}=\beta_{2 m}=p^{k+m-1}, \\
\gamma_{2 m+1}=\alpha_{1}=p^{k+m+r-r}, \quad \ldots, & \gamma_{2 m+r}=\alpha_{r}=p^{k+m+r-1}
\end{array}
$$

являются параметрами Сатаке рядов Эйзенштейна-Зигеля $F=E_{k+m+r}^{2 m+r}$.

ГиПотезА II (о подъеме второго рода из $\mathrm{GSp}_{r} \times \mathrm{GSp}_{2 m}$ в $\mathrm{GSp}_{r+2 m}$ ). Пусть

$$
(f, g) \in \mathscr{M}_{k+r-m}\left(\Gamma_{r}\right) \times \mathscr{M}_{k+r+m}\left(\Gamma_{2 m}\right)
$$

- пара собственных форм относительно алгебр Гекке $\mathscr{L}_{r}=\bigotimes_{p} \mathscr{L}_{r, p}$ u $\mathscr{L}_{2 m}=$ $\bigotimes_{p} \mathscr{L}_{2 m, p}$. Обозначим через $\alpha_{0}, \alpha_{1}, \ldots, \alpha_{r}$ u $\beta_{0}, \beta_{1}, \ldots, \beta_{2 m}$ p-параметры Сатаке борм $f$ и $g$ (вновь напомним, что эти параметры определены с точностью до действия группы Вейля).

Тогда существует собственнал форма $F$ относительно алгебры Гекке $\mathscr{L}_{r+2 m}$ рода $r+2 m$ и веса $k$ с параметрами Сатаке

$$
\gamma_{0}=\alpha_{0} \beta_{0}, \quad \gamma_{1}=\alpha_{1}, \quad \gamma_{2}=\alpha_{2}, \quad \ldots, \quad \gamma_{r}=\alpha_{r}, \quad \gamma_{r+1}=\beta_{1}, \quad \ldots, \quad \gamma_{2 m+r}=\beta_{2 m}
$$

для подходящего выбора $\alpha_{0}, \alpha_{1}, \ldots, \alpha_{r} u \beta_{0}, \beta_{1}, \ldots, \beta_{2 m}$ р-параметров Сатаке форм $f$ $u g$.

Первый пример выполнимости этой гипотезы дается второй формой Мияваки $F_{14}$, характеризуемой равенством [18; следствие 6.2, с. 333]

$$
L\left(F_{14}, \mathrm{St}, s\right)=L(f \otimes f, s+11) L(\varphi, s+13) L(\varphi, s+12), \quad \text { где } \quad f=\Delta, \quad \varphi=g_{26},
$$

так что при $m=r=1, k=12, f=\Delta \in S_{k+r-m}\left(\Gamma_{r}\right)=S_{12}\left(\Gamma_{1}\right), g=F_{2}(\varphi) \in$ $S_{k+r+m}\left(\Gamma_{2 m}\right)=S_{14}\left(\Gamma_{2}\right)$ выполнено

$$
\begin{aligned}
& (f, g)=\left(f, F_{2 m}(\varphi)\right) \mapsto \mathscr{F}_{\varphi, f} \in S_{k+r+m}\left(\Gamma_{r+2 m}\right), \\
& (f, g)=\left(f, F_{2 m}(\varphi)\right) \in S_{k+r-m}\left(\Gamma_{r}\right) \times S_{k+r+m}\left(\Gamma_{2 m}\right) .
\end{aligned}
$$

Еще один пример выполнимости этой гипотезы (для любого $r$ ) приходит из рядов Зигеля-Эйзенштейна

$$
f=E_{k+r-m}^{r}, \quad g=E_{k+r+m}^{2 m}
$$

родов $r$ и $2 m$ и весов $k+r-m$ и $k+r+m$ соответственно: здесь мы имеем

$$
\begin{aligned}
& \alpha_{0}=1, \quad \alpha_{1}=p^{k-m+r-r}, \quad \ldots, \quad \alpha_{r}=p^{k-m+r-1}, \\
& \beta_{0}=1, \quad \beta_{1}=p^{k+m+r-2 m}, \ldots, \quad \beta_{2 m}=p^{k+m+r-1},
\end{aligned}
$$

откуда видно, что

$$
\begin{gathered}
\gamma_{0}=1, \quad \gamma_{1}=\alpha_{1}=p^{k+r+m-(r+2 m)}, \quad \ldots, \quad \gamma_{r}=\alpha_{r}=p^{k-m+r-1}, \\
\gamma_{r+1}=\beta_{1}=p^{k+m+r-2 m}, \quad \ldots, \quad \gamma_{2 m+r}=\beta_{2 m}=p^{k+m+r-1}
\end{gathered}
$$

являются параметрами Сатаке рядов Эйзенштейна-Зигеля $F=E_{k+m+r}^{2 m+r}$. 
Отметим красивое обсуждение частного случая $r=1$ этой гипотезы II в работе [19], а также его совместимости с гипотезой Делиня и другими результатами (например, гамма-множителями для функционального уравнения).

ЗАмЕчАниЕ. Если проанализировать спинорные $L$-функции в этих гипотезах (задаваемые параметрами Сатаке

$$
\begin{gathered}
\gamma_{0}=\alpha_{0} \beta_{0}, \quad \gamma_{1}=\beta_{1}, \quad \gamma_{2}=\beta_{2}, \quad \ldots, \quad \gamma_{2 m}=\beta_{2 m}, \\
\gamma_{2 m+1}=\alpha_{1}, \quad \ldots, \quad \gamma_{2 m+r}=\alpha_{r}
\end{gathered}
$$

в гипотезе I, или же

$$
\gamma_{0}=\alpha_{0} \beta_{0}, \quad \gamma_{1}=\alpha_{1}, \quad \gamma_{2}=\alpha_{2}, \quad \ldots, \quad \gamma_{r}=\alpha_{r}, \quad \gamma_{r+1}=\beta_{1}, \quad \ldots, \quad \gamma_{2 m+r}=\beta_{2 m}
$$

в гипотезе II при подходящем выборе $\alpha_{0}, \alpha_{1}, \ldots, \alpha_{r}$ и $\beta_{0}, \beta_{1}, \ldots, \beta_{2 m}$ параметров Сатаке форм $f$ и $g$ (определенных с точностью до действия группы Вейля)), мы увидим, что эта спинорная $L$-функция соответствует тензорному произведению спинорных $L$-функций, и эта $L$-функция отличается от той, которая появляется в подъеме Иосиды [20], где $L$-функция сводится к произведению $L$-функций Гекке.

В этом контексте мы хотели бы отметить принцип функториальности Ленглендса: знаменатели наших $L$-рядов имеют вид локальных $L$-множителей Ленглендса (отвечающих представлениям двойственнй группы Ленглендса). Если рассмотреть гомоморфизмы

$$
\begin{aligned}
{ }^{L} \mathrm{GSp}_{r}=\operatorname{GSpin}(2 r+1) & \rightarrow \mathrm{GL}_{2^{r}}, \quad{ }^{L} \operatorname{GSp}_{2 m}=\mathrm{GSpin}(4 m+1) \rightarrow \mathrm{GL}_{2^{2 m}}, \\
{ }^{L} \mathrm{GSp}_{r+2 m} & =\mathrm{GSpin}(2 r+4 m+1) \rightarrow \mathrm{GL}_{2^{r+2 m}},
\end{aligned}
$$

то мы увидим, что наша гипотеза совместна с гомоморфизмом $L$-групп

$$
\mathrm{GL}_{2^{r}} \times \mathrm{GL}_{2^{2 m}} \rightarrow \mathrm{GL}_{2^{r+2 m}}, \quad\left(g_{1}, g_{2}\right) \mapsto g_{1} \otimes g_{2}, \quad \mathrm{GL}_{n}(\mathbb{C})={ }^{L} \mathrm{GL}_{n} .
$$

Очень приятно поблагодарить Стефена Гельбарта, Фрейдуна Шахиди, Жака Тилуина и Михаила Цфасмана за ценные обсуждения и наблюдения.

Выражаю особую благодарность А. Кузнецову и А. Парштну за организацию моего доклада на семинаре И. Р. Шафаревича 3 марта 2009 г., а также Ирине Резвяковой за предложение подготовить статью для специального тома "Математических заметок", посвященного памяти Анатолия Алексеевича Карацубы.

\section{СПИСОК ЦИТИРОВАННОЙ ЛИТЕРАТУРЫ}

[1] М. М. Вишик, Ю. И. Манин, "p-адические ряды Гекке мнимых квадратичных полей”, матем. сб., 95:3 (1974), 357-383.

[2] N. M. Katz, "p-Adic L-functions for CM-fields", Invent. Math., 49:3 (1978), 199-297.

[3] J.-P. Serre, "Formes modulaires et fonctions zêta $p$-adiques", Modular Functions of one Variable, III, Lecture Notes in Math., 350, Springer-Verlag, Berlin, 1973, 191-268.

[4] A. Weil, "On a certain type of characters of the idèle-class group of an algebraic number-field", Proceedings of the International Symposium on Algebraic Number Theory (Tokyo \& Nikko, 1955), Sci. Council of Japan, Tokyo, 1956, 1-7. 
[5] M. Deuring, "Die Zetafunktion einer algebraischen Kurve vom Geschlechte Eins", Nachr. Akad. Wiss. Göttingen. Math.-Phys. Kl. Math.-Phys.-Chem. Abt., 1953, 85-94; "Die Zetafunktion einer algebraischen Kurve vom Geschlechte Eins. II", Nachr. Akad. Wiss. Göttingen. Math.-Phys. Kl. Math.-Phys.-Chem. Abt., 1955, 13-42; "Die Zetafunktion einer algebraischen Kurve vom Geschlechte Eins. III", Nachr. Akad. Wiss. Göttingen. Math.-Phys. Kl. Math.-Phys.-Chem. Abt., 1956, 37-76; "Die Zetafunktion einer algebraischen Kurve vom Geschlechte Eins. IV", Nachr. Akad. Wiss. Göttingen. Math.-Phys. Kl. Math.-Phys.-Chem. Abt., 1957, 55-80.

[6] H. Hida, Elementary Theory of L-Functions and Eisenstein Series, London Math. Soc. Stud. Texts, 26, Cambridge Univ. Press, Cambridge, 1993.

[7] А.Н. Андрианов, "Эйлеровы произведения, отвечающие модулярным формам Зигеля рода 2", УМН, 29:3 (1974), 43-110.

[8] E. Hecke, "Über Modulfunktionen und die Dirichletschen Reihen mit Eulerscher Produktentwicklung. I. II", Math. Ann., 114:1 (1937), 1-28; "Über Modulfunktionen und die Dirichletschen Reihen mit Eulerscher Produktentwicklung. II", Math. Ann., 114:1 (1937), 316-351.

[9] M. Asgari, R. Schmidt, "Siegel modular forms and representations", Manuscripta Math., 104:2 (2001), 173-200.

[10] R.A. Rankin, "Contribution to the theory of Ramanujan's function $\tau(n)$ and similar arithmetical functions. I. The zeros of the function $\sum_{n=1}^{\infty} \tau(n) / n^{s}$ on the line $\Re s=13 / 2$ ", Proc. Camb. Phil. Soc, 35 (1939), 351-356; "Contribution to the theory of Ramanujan's function $\tau(n)$ and similar arithmetical functions. II. The order of the Fourier coefficients of integral modular forms", Proc. Camb. Phil. Soc, 35 (1939), 357-372.

[11] G. Shimura, "On the Holomorphy of certain Dirichlet Series", Proc. London Math. Soc. (3), 31:1 (1975), 79-98.

[12] А.Н. Андрианов, В.Л. Калинин, “Об аналитических свойствах стандартных дзета-функций зигелевых модулярных форм”, Матем. сб., 106:3 (1978), 323-339.

[13] S. Böcherer, "Über die Funktionalgleichung Automorpher $L$-Funktionnen zur Siegelschen Modulgruppe", J. Reine Angew. Math., 362 (1985), 146-168.

[14] T. Ikeda, "On the lifting of elliptic cusp forms to Siegel cusp forms of degree $2 n$ ", Ann. of Math. (2), 154:3 (2001), 641-681.

[15] T. Ikeda, "Pullback of the lifting of elliptic cusp forms and Miyawaki's Conjecture", Duke Math. J., 131:3 (2006), 469-497.

[16] K. Murakawa, "Relations between symmetric power $L$-functions and spinor $L$-functions attached to Ikeda lifts", Kodai Math. J., 25:1 (2002), 61-71.

[17] H. Kawamura, Congruences for the Ikeda lifts of Hida's families mod 11, 19 and 23, http://www-fourier.ujf-grenoble.fr/ panchish/KawamuraCong11-19-23.pdf.

[18] I. Miyawaki, "Numerical examples of Siegel cusp forms of degree 3 and their zeta-functions", Mem. Fac. Sci. Kyushu Univ. Ser. A, 46:2 (1992), 307-339.

[19] B. Heim, Towards Functoriality of Spinor L-Functions, Manuscript, MPIM, Bonn, 2008, $18 \mathrm{pp}$.

[20] H. Yoshida, "Siegel's modular forms and the arithmetic of quadratic forms", Invent. Math., 60:3 (1980), 193-248.

\section{А. А. Панчишкин}

Поступило

Institut Fourier, Université Grenoble-1

30.11 .2009

E-mail: panchish@ujf-grenoble.fr 